\author{
EVA TESHAJEV SUNDERLAND \\ Baltische Föderale Immanuel-Kant-Universität Kaliningrad \\ eteshajev@gmail.com \\ ORCID: 0000-0003-2202-548X
}

\title{
Der Einsatz von kurzen lustigen Videos im DaF-Unterricht. Möglichkeiten für die Wortschatz- und Grammatikarbeit
}

\author{
The use of short and funny videos in German \\ language lessons: Possibilities for teaching \\ of vocabulary and grammar
}

\begin{abstract}
Because emotions play a major role in the learning and memorising process, it is of high importance to create positive emotions in order to make language learning more efficient and enjoyable. The aim of this article is to show how a very popular source of entertainment, funny animal videos, can be used to make language learning more attractive to students. The author explores the connections between emotions - such as fun, joy and curiosity - and brain activity during learning. She then describes a range of exercises based on funny animal videos which can be used to introduce and practice vocabulary and grammar at different language levels and to create positive associations with the foreign language, in this case German.
\end{abstract}

KEYWORDS: Positive emotions and their influence on learning and remembering, videos in language teaching, motivation and optimal learning situations, exercises for learning vocabulary and grammar.

SCHLÜSSELWORTE: Positive Emotionen und ihr Einfluss auf Lernen und Erinnerung, Videos im Sprachunterricht, Motivation und optimale Lernsituation, Übungen zur Wortschatz- und Grammatikarbeit. 


\section{EINLEITUNG}

In unserem modernen Internetzeitalter, in dem die Aufmerksamkeitsspanne gering ist, wird das Schauen von Videos, in der Art wie man sie z.B. auf Youtube findet, immer verbreiteter. Insbesondere lustige Tiervideos erfreuen sich großer Beliebtheit, zahlreichen Menschen werden solche Videos von Freunden oder Bekannten zugeschickt, etliche Internetbenutzer verbringen viel Zeit mit dem Ansehen dieser Videobeiträge auf Youtube. Der israelische Historiker Yuval Noa Harari (2018) machte in einem Interview mit der Zeitschrift Stern sogar auf die Probleme aufmerksam, die seiner Ansicht nach mit putzigen Tiervideos verbunden sind:

Ich mag keine Katzenvideos - so viel Zeit wird an Katzenvideos vergeudet. [...] All diese Dinge funktionieren, weil sie sich in die Prozesse unseres Gehirns hacken. Sie wurden von Experten entwickelt, die sich wirklich gut mit den Mechanismen menschlicher Psychologie und Hirnforschung auskennen. In den letzten 20 Jahren haben sich die besten Leute auf diesem Gebiet damit beschäftigt, wie man jemanden verführt, etwas anzuklicken oder mehr Zeit mit dem Smartphone zu verbringen ${ }^{1}$.

Es ist erwiesen, dass die Videos, auf die sich Harari bezieht, millionenfach angeklickt werden. Zum Vergleich sei hier die Zahl der Aufrufe eines beliebten Youtube-Beitrags zum Erlernen von Deutsch als Fremdsprache angeführt: Der Beitrag „Aktiviere endlich dein Deutsch!“2 erzielte vom 5.05.2017 bis zum 4.11.2018 425.856 Aufrufe, ein Video, in dem Tieren lustige Missgeschicke passieren (Try Not To Laugh Challenge - Funny Cat \& Dog Vines compilation 2017)3, wurde hingegen im Zeitraum vom 18.11.2017 bis zum 4.11.2018 über 107 Mio. Male (107.257.827) aufgerufen.

Da die Videos das Bedürfnis der Menschen erfüllen Lustvolles zu erleben, könnte man sie auch nutzen, um das Angenehme mit dem Sinnvollen zu verknüpfen - z.B. mit dem Erlernen einer Fremdsprache, wie etwa des Deutschen, das bisweilen als „Hürdenlauf über grammatikalische Schwierigkeiten" bezeichnet wird (Picht 1980: 128).

Der vorliegende Beitrag befasst sich mit der Frage, wie die Popularität der oben genannten Videos genutzt werden kann, um den DaF-Unterricht lustvoller und effektiver zu gestalten und positive Assoziationen mit Spra-

\footnotetext{
1 Stern-Gespräch: „Wissenschafts-Weltstar Harari: ,Wir sollten die menschliche Dummheit nie unterschätzen'“. https://www.stern.de/panorama/weltgeschehen/yuval-noah-harari---diemenschliche-dummheit-nie-unterschaetzen--8358082.html [23. September 2018] [Zugriff am 7.11.2018].

${ }^{2}$ https:/ / www.youtube.com/watch?v=lK-y9WwhH2g [Zugriff am 7.11.2018].

${ }^{3}$ https://www.youtube.com/watch?v=EtH9Yllzjcc [Zugriff am 7.11.2018].
} 
che und Grammatik zu erzeugen. Zunächst wird erläutert, wie lustvolles Erleben Lernen und Gedächtnis beeinflusst. Dabei werden auch die besonderen Vorteile von Tiervideos im Vergleich zu anderem Videomaterial beleuchtet. Anschließend wird anhand einer Reihe von Beispielen veranschaulicht, wie lustige Tiervideos als regelrechte „Fundgrube“ für die Didaktisierung von Wortschatz und Grammatik genutzt werden können.

\section{POTENZIELLE WIRKUNGEN VON LUSTIGEN TIERVIDEOS UND IHR EINFLUSS AUF DAS LERNEN}

Die Arbeit mit Videos im DaF-Unterricht ist in verschiedener Hinsicht sinnvoll, da sie die Aktivität und Kreativität fördert (vgl. Brandi 2000: 35). Videobeiträge, in denen Tiere tollpatschiges oder auch menschenähnliches Verhalten an den Tag legen, erzeugen überdies bei den meisten Zuschauern positive Emotionen, wie gute Laune, Freude, Entspannung, Neugier. Welcher Zusammenhang besteht aber zwischen Emotionen und dem Lernprozess?

Gefühl und Denken sind auf das Engste miteinander verbunden, und stehen in ständigen Wechselwirkungen zueinander (vgl. Spitzer 2007: 167; Ciompi 1997). Roth (2001: 291) hebt die Rolle der Emotionen bei der Steuerung von Gedanken, Vorstellungen und der Erinnerung hervor. Einmal durchlebte Emotionen in Zusammenhang mit einem neu erlernten Sachverhalt beeinflussen unsere spätere Einstellung zu diesem Sachverhalt. Ciompi (1997) weist darauf hin, dass sogar bei der Speicherung einer neuen Wegstrecke Emotionen eine wichtige Rolle spielen, denn die

rein kognitiven Kennzeichen dieses Weges werden [auch mit] Angst- oder Lustgefühle[n] im Gedächtnis [...] behalten, die mit drohenden Gefahrenquellen oder mit Orten verbunden sind, die [...] Schutz bieten. Damit werden situationsgerechte psychische Gestimmtheiten und Verhaltensweisen wie Vorsicht, gespannte Aufmerksamkeit oder Entspannung mitsamt den zugehörigen körperlichen Bereitschaften bei jedem erneuten Begehen desselben Weges quasi automatisch reaktiviert (Ciompi 1997: 47).

Auch wenn jemand sich eines wiederholten gewohnten Reizes nicht mehr bewusst ist, wird von diesen Reizen dennoch eine entsprechende Grundstimmung ausgelöst (Ciompi 1997: 103).

Für den Fremdsprachenunterricht bedeutet dies, dass frühere negative oder positive Lernerfahrungen sich auf die Einstellung gegenüber der Sprache auswirken können, da mit ihr angenehme oder unangenehme Assoziationen verbunden werden. 
Ciompi, der das Konzept der Affektlogik entwickelte, demzufolge „emotionale und kognitive Komponenten - oder Fühlen und Denken, Affekte und Logik - in sämtlichen psychischen Leistungen untrennbar miteinander verbunden [sind] und gesetzmäßig zusammen [wirken]“ (Ciompi 1997: 46), teilt den einzelnen (Grund-)Affekten bestimmte "spezifische Operatorwirkungen auf Denken und Verhalten" zu:

- Interesse (bzw. Neugier, "Stimulushunger") weckt die Aufmerksamkeit (arousal) und lenkt sie auf bestimmte kognitive Entitäten.

- Angst bewirkt Abwendung, Distanzierung und evtl. Flucht vor bestimmten, als gefährlich konnotierten Kognitionen.

- Aggressive Gefühle wie Wut und Hass aktivieren Abwehr oder Angriff gegen bestimmte kognitive Entitäten [...].

- „Positive“ Gefühle wie Freude, Lust, Liebe, Vertrauen gehen mit Zuwendung, Annäherung und Bindung an bestimmte kognitive Entitäten einher" (Ciompi 2005: 53).

Positive bzw. lustbetonte Gefühle schaffen also Bindungen und diese Bindungen beziehen sich nicht nur auf Menschen, sondern auch auf unbelebte Objekte, geistige Gebilde und somit auch auf Sprachen. $\mathrm{Zu}$ solchen kognitiven Entitäten, mit denen man lustvolle Erfahrungen gemacht hat, fühlt man sich hingezogen, man sucht ihre Nähe (Ciompi 1997: 102). Weiterhin fühlt man sich dazu "motiviert" (bewegt), den Kontakt mit der attraktiven kognitiven Entität aufrechtzuerhalten, denn schon Freud wies darauf hin, dass ,jede einmal erfahrene Lust [...] nach Wiederholung [drängt]“ (Freud, nachfolgend Ciompi 1997: 111). Dies sind Erkenntnisse, die man auch im Bereich des Fremdsprachenerwerbs häufig erfahren kann, denn immer wieder gibt es Menschen, die ihre Motivation zum Erlernen einer fremden Sprache damit begründen, dass sie die Sprache um ihres schönen Klangs willen erlernen, der in ihnen positive Gefühle hervorruft, bzw. dass ihnen das Sprechen und Hören dieser Sprache Freude bereitet, auch wenn sie es nicht genauer erklären können. Bei Weitem nicht alle Lernenden beginnen jedoch ihren Spracherwerb mit solch einer positiven Grundhaltung, denn viele sind von Nützlichkeits- oder Kommunikationsmotiven angetrieben, andere wiederum erlernen die Fremdsprache aus einem Zwang heraus. Gerade für solche Lernenden ist es somit von großer Bedeutung Lernsituationen zu schaffen, in denen sie positive Emotionen erleben, dadurch positive Assoziationen zu Sprache und Lerninhalt entwickeln und sich schließlich zum Unterricht „hingezogen“ fühlen, also ihn gerne besuchen.

Während somit Angst und Aggression eine destruktive Wirkung auf das Lernen ausüben und daher vermieden werden sollten, machen positive Gefühle die Lernenden empfänglicher für neue Informationen oder wie der Gehirnforscher Prof. Dr. Manfred Spitzer (2007: 167) es ausdrückt: „Lernen 
[funktioniert] bei guter Laune am besten“. Spitzer führte eine Untersuchung durch, in der gezeigt werden konnte, dass beim Erlernen von Wörtern in einem positiven emotionalen Kontext der Bereich des Hippokampus und Parahippokampus aktiv ist, während beim Einspeichern von Wörtern in einem negativen emotionalen Kontext die Amygdala aktiviert wird. Diese Aktivierung unterschiedlicher Gehirnbereiche beeinflusst erfolgreiches Lernen, da sie mit der späteren Erinnerungsleistung in Zusammenhang steht. So konnte nachgewiesen werden, dass die ProbandInnen sich am besten an Wörter erinnern konnten, deren Einspeicherung in positivem emotionalem Kontext erfolgte (Spitzer 2007: 165-166). Heutzutage könnte man zwar annehmen, dass die Notwendigkeit des Schaffens von positiven Lernsituationen eine Selbstverständlichkeit ist, nichtsdestotrotz wird immer noch allzu oft der emotionale Aspekt des Lernens vernachlässigt, sodass es m. E. wichtig ist, die Verbindung von Denken und Fühlen wiederholt zu betonen.

Neben den positiven Gefühlen kann ebenfalls der weiter oben im Zusammenhang mit Ciompis Affektlogik erwähnte (Grund-)Affekt des Interesses bzw. der Neugier durch Videobeiträge hervorgerufen werden, sobald die Lernenden wissen, dass sie ein Video sehen werden, dessen Inhalt bzw. Thema sie noch nicht kennen. Das Zeigen von Videos ist ohnehin laut der Erkenntnisse der Motivationspsychologie für eine optimale Lernsituation förderlich, da es Neuigkeit, Überraschungsgehalt und Ungewissheit mit sich bringt (Kleppin 2002: 28). Der Überraschungsgehalt wird bei lustigen Tiervideos noch dadurch gesteigert, dass die Lernenden Videos solcher Art im DaF-Unterricht wohl kaum erwarten. Dadurch werden Interessegefühle erzeugt und diese „dienen in erster Linie der allgemeinen psychophysischen Anregung [...] und damit indirekt auch der Fokussierung der Aufmerksamkeit. [...] Gleichzeitig stellen sie den ganzen Körper auf Aktivität ein“" (Ciompi 1997: 100). Die geweckte Neugier hilft somit zusätzlich, sich Informationen zu dem entsprechenden Thema besser einzuprägen. Einer an der University of California at Davis durchgeführten Untersuchung zufolge, konnten sich die ProbandInnen Antworten auf bestimmte Fragen besser merken, wenn sie neugierig auf die Antwort waren. In dieser neugierigen Grundstimmung war ihre Aufmerksamkeit in so einem Maße aktiv, dass sie sich sogar zusätzliche Informationen merken konnten, die mit dem eigentlichen Thema nichts zu tun hatten und zwar über einen längeren Zeitraum. Die Aktivität des Hypocampus war sehr hoch. Durch die Neugier entsteht eine starke Interaktion zwischen Hypocampus und Belohnungssystem, so dass man einen Zustand erreicht, indem man auch nebensächliche Informationen besser behalten kann (Gruber 2018).

Abgesehen von der Tatsache, dass lustige Tiervideos positive Gefühle und Neugier wecken können, haben sie häufig einen weiteren Vorteil, der 
sie von anderem Videomaterial unterscheidet - die Niedlichkeit. In der Werbung ist die Wirkung von Niedlichkeit längst erkannt worden und so werden Tierbilder oder -aufnahmen vom sog. "cute marketing“ (niedlichem Marketing) als Aktivierungselement eingesetzt. Dem "cute marketing“ liegt das Kindchenschema zugrunde, welches bewirkt, dass bestimmte äußere Merkmale wie großer Kopf, runde Augen und kleine Nasen die Aufmerksamkeit des Betrachters erregen, das Gefühl der Fürsorge in ihm hervorrufen, oft ein Lächeln auf seinem Gesicht verursachen und das Belohnungssystem in seinem Gehirn aktivieren. Da Lebewesen, die Merkmale des Kindchenschemas aufweisen, das Kind im Betrachter ansprechen, bewirkt ihr Anblick in Stresssituationen eine Reduktion der mit dem Stress verbundenen negativen Gefühle (Brashear Alejandro, Granot \& Russell 2014: 75).

Amerikanische und japanische Studien belegen weitere wichtige Wirkungen des Betrachtens von Tierbildern bzw. - aufnahmen. So fanden japanische Forscher heraus, dass ihre ProbandInnen nach dem Betrachten der Fotos von Welpen und Kätzchen eine bessere Konzentrationsfähigkeit und einen erhöhten Aufmerksamkeitsfokus aufwiesen, was ihre systematische Verarbeitung von Informationen erhöhte und sie veranlasste im Rahmen von drei anschließenden Experimenten bei denen motorische, visuelle und deduktive Fähigkeiten getestet wurden, bessere Ergebnisse zu erzielen (Nittono, Fukushima, Yano \& Moriya 2012).

Die amerikanische Kommunikationsforscherin Jessica Gall Myrick wiederum kam in einer Untersuchung zu dem Schluss, dass das Sehen von lustigen Katzenvideos den Energiepegel ihrer ProbandInnen anhob. Laut Myrick handelt es sich beim Sehen solcher Videos keineswegs um Zeitvergeudung, da der zeitliche Aufwand sich auszahlt und den ProbandInnen im Anschluss hilft anspruchsvollere Aufgaben leichter zu bewältigen 4 .

Die Stressreduktion, Verbesserung der Aufmerksamkeit und der erhöhte Energiepegel die durch das Betrachten von Tierbildern bzw. -aufnahmen hervorgerufen werden, können wiederum sinnvoll im Sprachunterricht genutzt werden. Hierzu eignen sich Videos mit jeglichen Tieren, die Merkmale des Kindchenschemas aufweisen, gleichgültig ob es sich um Katzen, Hunde, Papageien oder andere handelt.

\footnotetext{
${ }^{4}$ Myrick äußerte sich dazu wie folgt: "Some people may think watching online cat videos isn't a serious enough topic for academic research, but the fact is that it's one of the most popular uses of the Internet today (...) If we want to better understand the effects the Internet may have on us as individuals and on society, then researchers can't ignore Internet cats anymore" (Not-so-guilty pleasure: Viewing cat videos boosts energy and positive emotions, IU study finds (2015). IU Bloomington Newsroom. Indiana University Bloomington. http:/ /archive.news. indiana.edu/releases/iu/2015/06/internet-cat-video-research.shtml [Zugriff am 30.05.2019].
} 


\section{VORSCHLÄGE ZUR DIDAKTISIERUNG}

Im Folgenden werden von der Verfasserin eingesetzte videobasierte Wortschatz- und Grammatikübungen vorgestellt, die sowohl im DaF-Unterricht mit jugendlichen als auch mit erwachsenen Lernenden zum Einsatz kamen und auf positives Feedback stießen, welches sich auch in messbaren Lernerfolgen widerspiegelte.

\subsection{Wortschatz - das bisschen Haushalt...}

Nützliche Ausdrücke und Vokabeln wie Geschirr spülen, Besen, aufräumen werden in Lehrbüchern häufig anhand von Bildern oder Zeichnungen veranschaulicht, was den Vorteil hat, dass die Lernenden das entsprechende Wort auch ohne Sprachkenntnisse verstehen und sich die fremdsprachlichen Wörter bildlich einprägen können. Allerdings ist das Thema Haushalt nicht für jeden Lernenden attraktiv und schematische Zeichnungen wirken auch nicht unbedingt anregend. Wie wirkt es aber, wenn alltägliche Haushaltstätigkeiten nicht von einem Menschen, sondern einem Tier ausgeführt werden? Zum einen findet man im Internet Fotos von Tieren, die sich im Haushalt versuchen und auf diese Weise bestimmte Tätigkeiten demonstrieren. Ein echter Waschbär, der mit einem Besen herumhantiert oder sich am Müllbeutel zu schaffen macht und eine Katze, die beim Staubsaugen assistiert, wecken erfahrungsgemäß das Interesse der Lernenden. Zum anderen gibt es auf Youtube eine Reihe von Videos, in denen dressierte Hunde mit Begeisterung verschiedene Aufgaben ausführen. Sie räumen unter anderem den Geschirrspüler ein, fegen oder wischen den Boden, bringen den Müll raus, machen Ordnung und putzen ihren Besitzern sogar die Schuhe. Der Anblick dieser Tätigkeiten fördert die Aufmerksamkeit des Zuschauers, denn dies sind keine Aufgaben, die man von einem Tier ohne Weiteres erwarten würde; und somit entsteht ein Überraschungseffekt (wie bei den Tierfotos auch). Ein großer Vorteil gegenüber den Fotos ist, dass es sich nicht um Momentaufnahmen handelt, sondern dass man auch den Bewegungsablauf beobachten kann, was die Situation einerseits lebendiger macht und anderseits die Konzentration der Lernenden fordert, denn es erweist sich, dass Hunde im Haushalt äußerst flink sind 5 .

\footnotetext{
${ }^{5}$ Hinzu kommt, dass sich die Tiere in realen Wohnräumen bewegen und somit - im Gegensatz zu schematischen Zeichnungen von Zimmern bzw. Wohnungen, die der Fantasie des Zeichners entspringen und oft gestellt wirken - ein zusätzlicher praktischer Bezug gegeben ist. Die Lernenden sehen so möglicherweise auch Details der Inneneinrichtung, deren fremdsprachliche Bezeichnung sie interessiert und nach der sie während der Lehrveranstaltung fragen können.
} 
Diese Videos ${ }^{6}$ lassen sich nun auf vielfältige Weise nutzen, so z.B. bei der Wiederholung des zuvor anhand der Fotos eingeführten Wortschatzes zum Thema Haushalt. Die Kursleiter können den Videobeitrag während jeder Tätigkeit des Hundes anhalten und die Lernenden im Präsens fragen „Was macht der Hund?". Die Lernenden antworten und üben gleichzeitig die Konjugation im Präsens („Der Hund geht einkaufen.“) sowie die Wortstellung bei trennbaren Verben („Der Hund sammelt die schmutzige Wäsche auf."). Weiterhin können die Kursleiter z.B. den Videobeitrag auch nach jeder erledigten Tätigkeit anhalten und nach den Perfektformen fragen („Was hat der Hund gemacht?).

Im Anschluss kann das Video als Gesprächsanregung für die Partnerarbeit dienen. Mögliche Fragen wären hierbei unter anderem:

Was hältst du von einer tierischen Haushaltshilfe?

Hättest du gerne tierische Unterstützung im Haushalt?

Welche Vor- und Nachteile hat ein Hund als Hilfe im Haushalt?

Welche Hausarbeiten magst du gar nicht? Warum?

\subsection{Grammatik}

\subsubsection{Das Passiv}

Mithilfe der weiter oben vorgestellten „Haushaltshund“-Videos lassen sich - nachdem der Wortschatz bereits bekannt ist - natürlich noch andere grammatische Formen einführen und veranschaulichen. Außer nach der Aktivform (Was macht der Hund? - Der Hund räumt das Zimmer auf.) lässt sich hierbei auch nach der Passivform fragen. Je nachdem wann das Video gestoppt wird - während oder nach den Tätigkeiten - kann entweder das Passiv im Präsens geübt werden:

Was wird gemacht?

Der Boden wird gewischt.

Oder man kann die Vergangenheitsformen des Passivs im Präteritum bzw. Perfekt abfragen:

Passiv Präteritum.

Was wurde gemacht?

Der Müll wurde rausgebracht.

${ }^{6}$ z.B. The Most Useful Dog Of The World. https://www.youtube.com/watch?v =x_FJt PSr_OU [Zugriff am 7.11.2018] und Trained Dog Cleans the House. https://www.youtube. com/watch?v=ro4nJIdpNgQ [Zugriff am 7.11.2018]. 


\section{Passiv Perfekt:}

Was ist gemacht worden?

Das Bett ist gemacht worden.

Eine mögliche Vorgehensweise für die Kursleiter besteht darin, zunächst anhand der ersten im Video gezeigten Tätigkeit die entsprechende Passivform zu erklären, sichtbar zu machen und anschließend die Lernenden aufzufordern, die nächsten beobachteten Haushaltsaufgaben selbst ins Passiv zu setzen.

Natürlich muss man nicht alle drei Formen auf einmal mit Hilfe eines solchen Videos einführen, um die Lernenden nicht zu überfordern. Da sich auf Youtube aber eine Reihe verschiedener Videos zu dem entsprechenden Thema findet, kann man als Kursleiter die Passivformen auf verschiedene Videos bzw. Lehreinheiten verteilen.

\subsubsection{Partizip I}

Klingelnde Telefone, hustende Menschen und weinende Babys haben alle die grammatische Form des Partizips I gemeinsam, stellen aber ansonsten nichts Außergewöhnliches dar. Außergewöhnlich wird die Sache hingegen, wenn ein Papagei das entsprechende Geräusch täuschend ähnlich nachahmt. Es versetzt uns immer wieder in Erstaunen, wozu das Sprachorgan dieses Vogels in der Lage ist und er ist zweifelsohne schöner anzuschauen, als der Rettungswagen, der Laserstrahl oder die Fernsprechanlage, die er imitiert. Amüsant wird es zudem, wenn er den Tobsuchtsanfall eines Kleinkindes nicht nur hervorragend akustisch, sondern auch gestisch nachahmt (z.B. indem er auf und ab hüpft). Somit lassen sich Papageien bestens für die Veranschaulichung und das Üben des Partizip I einspannen.

Eine Übung wie die folgende wäre unter Einsatz eines entsprechenden Videos ${ }^{7}$ denkbar:

Wen oder was ahmen die Papageien im Video nach?

Papagei 1 - Mensch - niesen (Antwort: Einen niesenden Menschen.)

Papagei 2 - Mann - husten (Antwort: Einen hustenden Mann.)

Papagei 3 - Frau - lachen (Antwort: Eine lachende Frau.)

${ }^{7}$ z.B. Funny Parrots Dance, Sing and Imitate Sounds - Funny Birds Meow, Bark, Cry, Phone \& Car Alarm Videos. https://www.youtube.com/watch?v=KIKh8wQX3FA [Zugriff am 7.11.2018]; Rockender Papagei. https://www.youtube.com/watch?v=eBgvC6wjREA [Zugriff am 7.11.2018]. 


\subsubsection{Substantive Verben}

Wenn das bitterliche Schluchzen eines Kleinkindes oder das schrille Klingeln eines Telefons einem Papageienschnabel entspringt, kann natürlich auch das substantivierte Verb mit den Lernenden geübt werden. Zum Beispiel auf folgende Weise:

Was ahmen die Papageien im Video 8 nach?

Papagei 1 - Baby - weinen (Antwort: Das Weinen eines Babys.)

Papagei 2 - Telefon - klingeln (Antwort: Das Klingeln eines Telefons.)

\subsubsection{Relativsätze}

Wie bereits gezeigt wurde, schlummern in Papageien unbekannte Talente, die sich für die Veranschaulichung vielfältiger Grammatikformen heranziehen lassen. Dazu gehören, neben dem Nachahmen der unglaublichsten Geräusche, auch hervorragendes Singen und Tanzen. Alle Aktivitäten eignen sich ebenfalls gut zum Üben von Relativsätzen. Möglich wäre eine Übung wie diese.

Was ist das für ein Papagei?

Ein Papagei, der einen Popsong singt ${ }^{9}$.

Ein Papagei, der Lambada tanzt ${ }^{10}$.

Aufgrund des Nachahmtalents diverser Youtube-Stars lässt sich die grammatische Form des Relativsatzes auch gut um neuen Wortschatz ergänzen:

Ein Papagei, der eine Katze nachahmt. (oder: Ein Papagei, der miaut wie eine Katze.)

Ein Papagei, der einen Hund nachahmt. (oder: Ein Papagei, der bellt wie ein Hund.)

Ein Papagei, der einen Hahn nachahmt. (oder: Ein Papagei, der kräht wie eine Hahn.)

${ }^{8}$ Funny Parrots Dance, Sing and Imitate Sounds - Funny Birds Meow, Bark, Cry, Phone \& Car Alarm Videos. https:/ / www.youtube.com/watch?v=KIKh8wQX3FA [Zugriff am 7.11.2018]; Rockender Papagei. https://www.youtube.com/watch?v=eBgvC6wjREA [Zugriff am 7.11.2018].

${ }^{9}$ Eminem's The Monster but Rihanna replaced with a Parrot. https://www.youtube. com/watch?v=I3CLgubUGo0 [Zugriff am 7.11.2018].

${ }^{10}$ Parrot Lambada Dancing. https://www.youtube.com/watch?v=FZ4SGukFE8w [Zugriff am 7.11.2018]. 
Ein Papagei, der einen Wolf nachahmt. (oder: Ein Papagei, der heult wie eine Wolf.)

Ein Papagei, der einen Tiger nachahmt. (oder: Ein Papagei, der knurrt wie eine Tiger.)

Und der weiter oben erwähnte beflügelte Lambadatänzer hat noch mehr zu bieten: So können fortgeschrittene Lernende mit Unterstützung der Kursleiter die Showeinlage des gefiederten Tanzstars beschreiben und somit den Wortschatz für Bewegungsabläufe üben (Er macht einen Schritt nach links/ nach vorne / nach hinten. Er dreht sich nach rechts. Er dreht sich um die eigene Achse. Er macht eine Pirouette. Er hebt seine Flügel an. Er wackelt mit den Hüften. Er wippt hin und her. Er läuft seitwärts. Er hüpft auf und ab. Er bewegt seinen Kopf im Takt. Er bewegt seinen Kopf vor- und rückwärts, seitwärts.). Diese Bewegungen können natürlich auch von Personen demonstriert werden, aber ein tanzender Mensch birgt lange nicht so viel Überraschungspotential wie ein hüftschwingender Kakadu mit Gefühl für Rhythmus.

\subsubsection{Perfektformen}

Sogar die berühmt-berüchtigten „Katzen und Gurken“-Videos"11, in denen sich Katzen fürchterlich vor dem Grünzeug erschrecken (und die man zuhause natürlich nicht nachstellen sollte) lassen sich für grammatische Zwecke heranziehen. So kann man die Lernenden im Perfekt berichten lassen, was sie im Video beobachten können:

Die Katze hat sich erschreckt.

Sie ist in die Luft gehüpft.

Sie hat die Futterschale umgeworfen.

Sie hat den Stuhl umgestoßen.

Sie ist aus der Küche gerannt.

Anschließend kann das Video als Redeanlass für die Partnerarbeit dienen, z.B. anhand der Fragen:

Wieso hat sich deiner Meinung nach die Katze so sehr vor der Gurke erschreckt?

Wann hast du dich das letzte Mal erschreckt? Wieso?

Hast du schon einmal jemanden erschreckt? Was ist passiert?

${ }^{11}$ Cats scared of Cucumbers Compilation - Cats Vs Cucumbers - Funny Cats. https:/ / www. youtube.com/watch?v=cNycdfFEgBc [Zugriff am 7.11.2018]. 


\section{SCHLUSSFOLGERUNGEN}

Zusammenfassend lässt sich sagen, dass lustige Tiervideos im Fremdsprachenunterricht vielfältig und auf allen Niveaustufen eingesetzt werden können. Sie wecken Neugier und Interesse, rufen Überraschung hervor und steigern die Aufmerksamkeit. Überdies erzeugen sie bei vielen Betrachtern positive Gefühle und können somit die Erinnerung nachhaltig beeinflussen. Somit haben sie das Potential, zu einer optimalen Lernsituation beizutragen.

Die Vorbereitung von Wortschatz- oder Grammatikübungen zu diesen Videos ist nicht zeitaufwendig, man muss nur seine Phantasie walten lassen. Durch Videos dieser Art lassen sich auch schwierigere oder weniger häufige Grammatikformen in einer angenehmen Weise einführen und üben, so dass der Deutschkurs statt zu einem - wie bereits in der Einleitung erwähnten „Hürdenlauf über grammatikalische Schwierigkeiten“ (Picht 1980: 128), zu einem Erlebnisort wird, mit dem man positive Assoziationen verknüpft, den man mit guter Laune verlässt und zu dem man gerne wiederkehrt. Schließlich gehört zu den Motiven von Fremdsprachenlernenden nach Apelt (1992: 88) nicht nur das Nützlichkeits- und Kommunikations-, sondern auch das Erlebnismotiv.

Hinzuzufügen ist allerdings, dass diese Methode nur dann im DaF-Unterricht eingesetzt werden sollte, wenn sie auch den Kursleitern zusagt und sie davon überzeugt sind. Eine etwaige Skepsis könnte sich sonst den Lernenden mitteilen und der Sinn der Übungen wäre verfehlt. Sind die Kursleiter aber von der Methode angetan, so können sie auch seine Begeisterung auf die Lernenden übertragen (Hüther 2004: 32-33).

Ferner gilt es seine Lerngruppe und ihre Einstellung zum Sprachunterricht richtig einzuschätzen, denn manchen Lernenden mag die vorgestellte Methode nicht ernsthaft genug erscheinen und auch dann würde sie nicht die erhoffte Wirkung erzielen.

Zuletzt sei noch angemerkt, dass auch ein zu häufiger Einsatz von Videos dieser Art - so beliebt sie auch sein mögen - vermieden werden sollte, da sie sonst nichts Neuartiges und Besonderes mehr darstellen. So weist z.B. Kleppin (2002: 28) darauf hin, dass neue und anregende Ressourcen nicht zu oft herangezogen werden sollten, da sonst eine Gewöhnung eintritt und die anregende Wirkung verloren geht.

Ein gelegentlicher Einsatz von derartigen Tiervideos hingegen macht die Lernsituation abwechslungsreicher und kann dazu beitragen, eine emotionale Bindung zu der zu erlernenden Fremdsprache herzustellen. Ein Ausprobieren ist somit auf jeden Fall empfehlenswert. 


\section{LITERATURVERZEICHNIS}

Apelt, W. (1992). Motive - Motivation - Motivierung. In: U. Jung (Hrsg.), Praktische Handreichungen für Fremdsprachenlehrer (S. 85-93). Frankfurt am Main: Peter Lang.

Brandi, M. (2000). Video im Deutschunterricht. Berlin: Langenscheidt.

Brashear Alejandro, T. / Granot, E. / Russell, L. T. M. (2014). A socio-marketing analysis of the concept of cute and its consumer culture implications. Journal of Consumer Culture, 14 (1), 66-87. https://www.researchgate.net/publication/270721118_A_socio-marketing_analysis_ of_the_concept_of_cute_and_its_consumer_culture_implications [Zugriff am: 30.05.2019].

Ciompi, L. (1997). Die emotionalen Grundlagen des Denkens. Entwurf einer fraktalen Affektlogik. Göttingen: Vandenhoeck \& Ruprecht.

Ciompi, L. (2005). Grundsätzliches zu Emotion, Kognition und Evolution aus der Humanperspektive. In: L. Ciompi / M. Wimmer (Hrsg.), Emotion - Kognition - Evolution. Biologische, psychologische, soziodynamische und philosophische Aspekte (S. 47-66). Fürth: Filander Verlag.

Gruber, M. J. / Gelman, B. D. / Ranganath, C. (2014). States of curiosity modulate hippocampus-dependent learning via the dopaminergic circuit. Neuron, 84, 486-496. http://dx.doi. org/10.1016/j.neuron.2014.08.060 [Zugriff am: 1.11.2018].

Harari, Y. N. (2018). Stern-Gespräch: Wissenschafts-Weltstar Harari: „Wir sollten die menschliche Dummheit nie unterschätzen“. Stern, 38. https://www.stern.de/panorama/weltge schehen/yuval-noah-harari---die-menschliche-dummheit-nie-unterschaetzen--8358082.html [23. September 2018] [Zugriff am 7.11.2018].

Hüther, G. (2004). Die Bedeutung emotionaler Reaktionen für Lernprozesse und die Verankerung neuer Erfahrungen. In: W. Börner / K. Vogel (Hrsg.), Emotion und Kognition im Fremdsprachenunterricht (S. 25-34). Tübingen: Gunter Narr Verlag.

IU Bloomington Newsroom (2015). Not-so-guilty pleasure: Viewing cat videos boosts energy and positive emotions, IU study finds. Indiana: Indiana Uniersity Bloomington. http://archive.news.in diana.edu/releases/iu/2015/06/internet-cat-video-research.shtml [Zugriff am: 30.05.2019].

Kleppin, K. (2002). Motivation. Nur ein Mythos? (II) Deutsch als Fremdsprache. Zeitschrift zur Theorie und Praxis des Deutschunterrichts für Ausländer, 39 (1), 26-30.

Nittono, H. / Fukushima, M. / Yano, A. / Moriya, H. (2012). The power of kawaii: Viewing cute images promotes a careful behavior and narrows attentional focus. PLoS ONE, 7 (9), 1-7. https://journals.plos.org/plosone/article?id=10.1371/journal.pone.0046362 [Zugriff am: 30.05.2019].

Picht, R. (1980). Interesse und Vergleich: zur Sozialpsychologie des Deutschlandbilds. In: A. Wierlacher et al., Jahrbuch Deutsch als Fremdsprache, 6 (S. 120-132). Heidelberg: Julius Groos.

Roth, G. (2001). Fühlen, Denken, Handeln. Wie das Gehirn unser Verhalten steuert. Frankfurt am Main: Suhrkamp.

Spitzer, M. (2007). Lernen. Gehirnforschung und die Schule des Lebens. Berlin, Heidelberg: Spektrum.

\section{VIDEOS}

Aktiviere endlich dein Deutsch!“. https://www.youtube.com/watch?v=1K-y9WwhH2g [Zugriff am 7.11.2018]. Cats scared of Cucumbers Compilation - Cats Vs Cucumbers - Funny Cats. https://www.youtube.com/watch?v=cNycdfFEgBc [Zugriff am 7.11.2018].

Eminem's The Monster but Rihanna replaced with parrot. https://www.youtube.com/ watch?v=I3CLgubUGo0 [Zugriff am 7.11.2018]. 
Funny parrots dance, sing and imitate sounds - funny birds meow, bark, cry, phone \& car alarm videos. https:/ / www.youtube.com/watch?v=K1Kh8wQX3FA [Zugriff am 7.11.2018].

The most useful dog of the world. https://www.youtube.com/watch?v=x_FJtPSr_OU [Zugriff am 7.11.2018].

Parrot Lambada dancing. https://www.youtube.com/watch?v=FZ4SGukFE8w [Zugriff am 7.11.2018].

Rockender Papagei. https://www.youtube.com/watch?v=eBgvC6wjREA [Zugriff am 7.11.2018].

Trained dog cleans the house. https://www.youtube.com/watch?v=ro4nJIdpNgQ [Zugriff am 7.11.2018].

Try not to laugh challenge - funny cat \& dog vines compilation 2017. https://www. youtube.com/watch?v=EtH9Yllzjcc [Zugriff am 7.11.2018].

Received: 2.03.2019; revised: 15.07.2019 